\title{
New Stability Criteria for Event-Triggered Nonlinear Networked Control System with Time Delay
}

\author{
Hongqian Lu $\mathbb{D}^{1},{ }^{1}$ Yue Hu, ${ }^{1}$ Chaoqun Guo, ${ }^{1}$ and Wuneng $\mathrm{Zhou}^{2}$ \\ ${ }^{1}$ School of Electrical Engineering and Automation, Qilu University of Technology (Shandong Academy of Sciences), \\ Jinan 250353, China \\ ${ }^{2}$ College of Information Science and Technology, Donghua University, Shanghai 201620, China
}

Correspondence should be addressed to Hongqian Lu; hqlu@163.com

Received 16 January 2019; Revised 21 April 2019; Accepted 19 May 2019; Published 10 June 2019

Academic Editor: Yan-Ling Wei

Copyright (C) 2019 Hongqian Lu et al. This is an open access article distributed under the Creative Commons Attribution License, which permits unrestricted use, distribution, and reproduction in any medium, provided the original work is properly cited.

\begin{abstract}
This note focuses on the stability and stabilization problem of nonlinear networked control system with time delay. To alleviate the burden of transformation channel and shorten the dynamic process simultaneously, an improved event-triggered scheme is proposed. This paper employs an improved time delay method to enhance the performance and reduce the delay upper bound conservatism. Less conservative stability criteria related to the order $N$ are derived by establishing an augmented LyapunovKrasovskii functional manufactured for the use of Bessel-Legendre inequality. In addition, an event-triggered controller is designed for nonlinear networked control system with time delay. At last, numerical examples are proposed to verify the effectiveness of the new method.
\end{abstract}

\section{Introduction}

In recent years, the networked control has been applied to the practical control process [1]. Compared with the point to point control method, the networked control system has better reliability and can reduce power requirements, operation cost $[2,3]$. There are different kinds of networked control systems, such as centralized networked control system [4], decentralized networked control system [5], distributed networked control system [6], and wireless networked control system [7]. In practice, the performance of networked control system is always influenced by the uncertainties and disturbance and nonlinear factors. Nonlinear networked control system's asymptotic behavior has been researched in [8]. Stability of nonlinear networked control system has been studied in [9]. The literature [10, 11] have investigated robust stability of nonlinear networked control system with uncertainties. This paper also takes into account nonlinearity to augment the performance of networked control system.

For traditional networked control system [12, 13], transmitting all sampled packets into the network is not always necessary from the point of view of the limited network channel resource under the time-triggered scheme. Thus, the event-triggered scheme (ETS) is proposed to reduce the burden of channel. Under the event-triggered condition, stochastic stability of nonlinear system was studied in [14]. The fault detection issue for nonlinear discrete-time networked systems was discussed in [15]. In recent years, researchers have improved event-triggered scheme continuously to adjust to various networked environments. A periodic ETS was proposed to overcome the shortcoming needing extra hardware to check triggering condition instantaneously [16]. For wireless sensor networks, the decentralized ETS was developed to better save the channel resource [17]. In order to shorten the dynamic process, an improved static ETS was researched in [18] which can increase the frequency of transmission at initial times. Furthermore, dynamic ETS was proposed by introducing a dynamic variable in triggering condition [19]. In this paper, we will put forward a new improved static ETS for the nonlinear networked control system with time delay to decrease the burden of channel and improve system dynamics.

At the same time, time delay problem has been widely investigated in the practical control system [20-24]. Variable time delay problems appear in control system [25-27]. For 
example, the stability problems of delay neural networks was studied in [28]. Based on the stochastic process, the random delay was researched in $[29,30]$. Robust $H_{\infty}$ stability of time delay system was researched widely [31-33]. Reference [34] studied a network-induced delay to deal with the network transmission delay problem. The distributed delay was developed for a class of neural network control system. However, in this improved event-triggered networked control system, the time delay problems still have a lot of room for improvement [35-38].

For the sake of reducing the conservatism of stability criterion of time delay systems, a series of technical approaches have been proposed. Before listing these approaches, we state that the conservatism of stability criteria mainly results from the estimation gap of the integral terms expressed as $\int_{t-d_{2}}^{t} \dot{x}^{T}(s) R \dot{x}(s) d s$ in the derivative of Lyapunov-Krasovskii functional. To study stability of system, model transformation approach was used in [39]. The stability criteria obtained by model transformation approach have large conservatism. To decrease the conservatism of stability criteria, a free weighting matrix method which can remedy the drawback of model transformation was proposed in [40]. However, free weighting matrix method will increase the decision variables, which makes the computation complex. To overcome this point and better estimate the integral terms, the Jensen inequality was used widely [41]. Afterwards, Wirtinger-based inequality which is considered a tighter method than Jensen inequality for estimation of the integral term was developed and employed in various systems [42]. In recent years, many researchers have improved the Wirtinger-based inequality approach, such as free-matrix-based integral inequality [43], auxiliary function-based integral inequality [44]. In this note, we will make use of a new integral inequality called Bessel-Legendre inequality together with reciprocally convex combination lemma to research the stability of nonlinear networked control system with time delay under improved event-triggered scheme.

As is well known, there is a quadratic integral term $\int_{t-d_{2}}^{t} \int_{\theta}^{t} \dot{x}^{T}(s) R \dot{x}(s) d s d \theta$ in Lyapunov-Krasovskii functional, which means that there will be a term $\int_{t-d_{2}}^{t} \dot{x}^{T}(s) R \dot{x}(s) d s$ in the derivative of Lyapunov-Krasovskii functional. We apply the Bessel-Legendre inequality to estimate $\int_{t-d_{2}}^{t} \dot{x}^{T}(s) R \dot{x}(s) d s$ and obtain that

$$
\int_{t-d_{2}}^{t} \dot{x}^{T}(s) R \dot{x}(s) d s \geq d_{2} \phi_{N}^{T} \bar{R}_{N} \phi_{N},
$$

where $\phi_{N}=\left(1 / d_{2}\right) \int_{-d_{2}}^{0} \mathbb{L}_{N}\left(\left(s+d_{2}\right) / d_{2}\right) x(s) d s, \bar{R}_{N}=\operatorname{diag}(R$, $3 R, \ldots,(2 N+1) R)$ and $\mathbb{L}_{N}$ is Legendre polynomial matrix. This inequality provides a tighter bound on this specific term, which makes the obtained stability condition less conservative. In addition, we will construct an appropriate Lyapunov-Krasovskii functional manufactured for the use of Bessle-Legendre inequality.

The main contributions of this paper are summarized as follows. Firstly, an improved ETS is put forward for nonlinear networked control system in this paper to reduce transmission load of channel by decreasing the number of signal transmission. The triggering parameter in this improved scheme is time-varying to achieve the situation that transmission frequency at the beginning instants is higher than at the other times, which can shorten the dynamic process of system effectively. Secondly, less conservative stability criteria subject to the order $N$ are obtained by employing the Bessel-Legendre inequality and introducing a Legendrebased Lyapunov-Krasovskii functional. Conservatism will be reduced with the increase of the $N$. Furthermore, a controller is designed for event-triggered nonlinear networked control system with time delay.

The rest of the paper is summarized as follows. Section 2 gives the considered nonlinear networked control system and puts forward an improved ETS. In Section 3, less conservative stability criteria are derived via Bessel-Legrendre inequality method. Section 4 designs a controller for the system in this paper. To verify the effectiveness of results, numerical examples are shown in Section 5. Finally, conclusions are summarized in Section 6.

Notations: In this paper, symbol $T$ denotes the transpose. The $\mathbb{R}^{n}$ denotes the $\mathrm{n}$-dimensional Euclidean space. $\mathbb{R}^{n \times m}$ is the set of all $n \times m$ matrices. The set $\mathbb{S}^{n}\left(\mathbb{S}_{+}^{n}\right)$ means the set of the symmetric (positive definite) matrices of $\mathbb{R}^{n \times n}$. Furthermore, $\mathrm{He}(A)=A+A^{T}$. For matrices $A \in \mathbb{R}^{u \times v}$ and $B \in \mathbb{R}^{m \times n}$, their Kronecker product is a matrix in $\mathbb{R}^{u m \times v n}$ denoted as $A \otimes B$. The $\mathbb{N}$ denotes non-negative integer. $I_{n}$ denotes the identity matrix with $n \times n$ dimensions. $I_{n N}$ denotes the identity matrix with $n(N+1) \times n(N+1)$ dimensions.

\section{Problem Formulation}

In this paper, it is assumed that the networked control system has nonlinear function which satisfies the Lipschitz condition and the system state is fully observable. Thus, in this section, we establish the system model as

$$
\begin{aligned}
& \dot{x}(t)=A x(t)+A_{\tau} f(x(t))+B u(t), \\
& z(t)=C x(t), \\
& x(t)=\phi(t), \quad t \in\left[-d_{2}, 0\right),
\end{aligned}
$$

where $x(t) \in \mathbb{R}^{n}$ is the state; $z(t) \in \mathbb{R}^{q}$ is the controlled output; $u(t) \in \mathbb{R}^{m}$ denotes the control input; $A, A_{\tau}, B, C$ are real constant matrices; $\phi(t)$ denotes the initial condition function; $d_{2}$ is a positive scalar. Furthermore, $f(x(t))$ is nonlinear function and satisfies

$$
|f(x)-f(y)| \leq|F(x-y)|,
$$

where $F$ is a known constant matrix.

In the nonlinear networked control system, there exists a phenomenon of transmitting some unnecessary sampling data during the transmission from the sensor to the controller. In order to improve the networked control system transmission performance, this paper will propose an improved event-triggered mechanism to reduce the load of the network transmission. Next, we will build an eventtriggered generator for the nonlinear networked control 


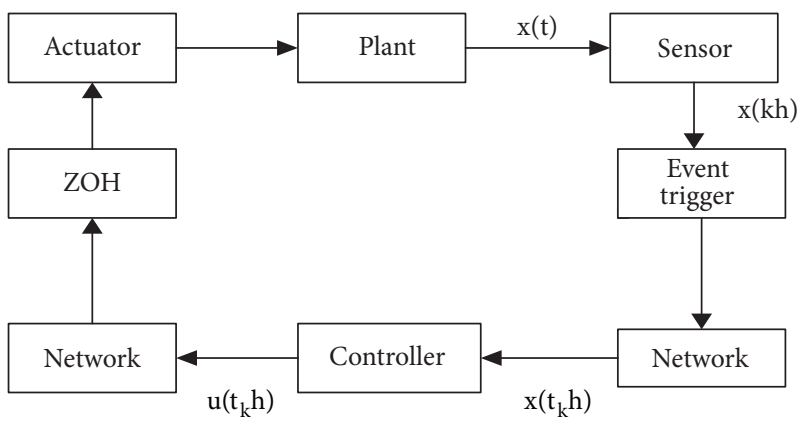

FIGURE 1: Schematic of networked control system with ETS.

system. It is assumed that the sampling sequence is $\mathbb{S}_{s}=$ $\{0, h, 2 h, \ldots, n h\}$. Suppose $t_{k} h$ is the current released time and $t_{k+1} h$ is the next released time. In addition, $t_{k+1} h=t_{k} h+n h$, where $n h$ is the release interval of the transmitted data.

Although the data is released at $t_{k} h$, it will arrive at actuator at $t_{k} h+\widehat{\tau}_{k}$ instant resulting from the existence of time delay $\widehat{\tau}_{k} \in[0, \bar{\tau}), k \in\{0,1,2, \cdots\}$, scalar $\bar{\tau}>0$.

Next, based on $[18,35]$ and the diagram of event-triggered networked control system in Figure 1, a network time delay model for the nonlinear networked control system can be constructed. Suppose that

$$
\begin{aligned}
\rho_{k} & =\min \left\{j \mid t_{k} h+\widehat{\tau}_{k}+j h \geq t_{k+1} h+\widehat{\tau}_{k+1}, j\right. \\
& =0,1,2, \ldots\} .
\end{aligned}
$$

The interval $\left[t_{k} h+\widehat{\tau}_{k}, t_{k+1} h+\widehat{\tau}_{k+1}\right)$ can be rewritten as

$$
\left[t_{k} h+\widehat{\tau}_{k}, t_{k+1} h+\widehat{\tau}_{k+1}\right)=\bigcup_{j=1}^{\rho_{k}} \Pi_{j}
$$

where

$$
\begin{aligned}
& \Pi_{j}=\left[t_{k} h+\widehat{\tau}_{k}+(j-1) h, t_{k} h+\widehat{\tau}_{k}+j h\right), \\
& j=1,2, \ldots, \rho_{k}-1, \\
& \Pi_{\rho_{k}}=\left[t_{k} h+\left(\rho_{k}-1\right) h+\widehat{\tau}_{k}, t_{k+1} h+\widehat{\tau}_{k+1}\right) \text {. } \\
& d(t)= \begin{cases}t-t_{k} h, & t \in \Pi_{1} \\
t-t_{k} h-h, & t \in \Pi_{2} \\
\vdots & \vdots \\
t-t_{k} h-\left(\rho_{k}-1\right) h, & t \in \Pi_{\rho_{k}}\end{cases} \\
& e_{k}(t)= \begin{cases}0, & t \in \Pi_{1} \\
x\left(t_{k} h\right)-x\left(t_{k} h+h\right), & t \in \Pi_{2} \\
\vdots & \vdots \\
x\left(t_{k} h\right)-x\left(t_{k} h+\left(\rho_{k}-1\right) h\right), & t \in \Pi_{\rho_{k}}\end{cases}
\end{aligned}
$$

where $0<\widehat{\tau}_{k} \leq d(t) \leq \bar{\tau}+h$. We set the $d_{2}=\bar{\tau}+h$, then $0<\widehat{\tau}_{k} \leq d(t) \leq d_{2}$.
For the $t \in\left[t_{k} h+\widehat{\tau}_{k}, t_{k+1} h+\widehat{\tau}_{k+1}\right)$, the event-triggered condition is

$$
e_{k}^{T}(t) \Omega e_{k}(t) \leq \sigma_{k}(t) x^{T}(t-d(t)) \Omega x(t-d(t)),
$$

$\Omega \in \mathbb{S}_{+}^{n}, \sigma_{k}(t)$ is a time-varying function as

$$
\sigma_{k}(t)=\sigma_{k j}, \quad t \in \Pi_{j}, j=1,2, \ldots, \rho_{k},
$$

where

$$
\begin{aligned}
\sigma_{k(j+1)} & =\bar{\sigma}+\frac{e_{k j}^{T} e_{k j}}{\lambda+e_{k j}^{T} e_{k j}}\left(\sigma_{k j}-\bar{\sigma}\right), \\
e_{k j} & =x\left(t_{k} h\right)-x\left(t_{k} h+(j-1) h\right), \\
& j=1,2, \ldots, \rho_{k},
\end{aligned}
$$

where known constants $\bar{\sigma} \in[0,1], \lambda>0,0<\sigma_{k 1}<\bar{\sigma}$, and $\sigma_{k 1}$ is the initial value of $\sigma_{k j}$. It is easy to see that the $\sigma_{k}(t)$ is monotonically increasing and has an upper bound $\bar{\sigma}$.

Remark 1. The sampled data will be transmitted when condition (9) is not satisfied. It is noticed that the $\sigma_{k}(t)$ in the improved event-triggered scheme (9) is time-varying and meets (10), which can increase the triggering time at the initial times to optimize the dynamic process of the system in this paper.

Therefore, according to formulae (2)-(9), we have

$$
u\left(t_{k} h\right)=K x\left(t_{k} h\right)=K e_{k}(t)+K x(t-d(t)),
$$

where $t \in\left[t_{k} h+\widehat{\tau}_{k}, t_{k+1} h+\widehat{\tau}_{k+1}\right), K \in \mathbb{R}^{m \times n}$ represents networked controller gain, then the system model can be rewritten as follows:

$$
\begin{aligned}
\dot{x}(t)= & A x(t)+A_{\tau} F x(t)+B K x(t-d(t)) \\
& +B K e_{k}(t), \\
z(t)= & C x(t), \\
x(t)= & \phi(t), \quad\left[-d_{2}, 0\right),
\end{aligned}
$$

where

$$
\begin{aligned}
0 & <\widehat{\tau}_{k} \leq d(t) \leq d_{2}, \\
d_{m} & \leq \dot{d}(t) \leq d_{M},
\end{aligned}
$$

$d_{m}, d_{M}$ are known constants, $d_{m}<0, d_{M}>0$.

For analyzing the stability and stabilization problem of nonlinear networked control system conveniently, we will give a definition and some lemmas.

Definition 2 (see [24]). For given scalars $i, j \in \mathbb{N}$, the Legendre polynomial considered over the interval $\mu \in[0,1]$ is

$$
L_{i}(\mu)=(-1)^{i} \sum_{j=0}^{i} p_{j}^{i} \mu^{j},
$$

where $p_{j}^{i}=(-1)^{j}\left(\begin{array}{c}i \\ j\end{array}\right)\left(\begin{array}{c}i+j \\ j\end{array}\right)$, and $\left(\begin{array}{l}k \\ l\end{array}\right)$ means $k ! /(k-l) ! l !$. 
Define that

$$
\mathbb{L}_{N}(\mu):=\left[L_{0}(\mu) I_{n}, \ldots, L_{N}(\mu) I_{n}\right]^{T}
$$

is a polynomial matrix with $(N+1) n \times n$ dimensions, where the integers $N \geq 0, n>0$.

Lemma 3 (see [28], reciprocally convex inequality). Let integer $n>0$ and $R_{1}, R_{2}$ be in $\mathbb{S}_{+}^{n}$. If there exist $X_{1}, X_{2}$ in $\mathbb{S}^{n}$ and $Y_{1}, Y_{2}$ in $\mathbb{R}^{n \times n}$ such that

$$
\left[\begin{array}{cc}
R_{1} & 0 \\
0 & R_{2}
\end{array}\right]-\alpha\left[\begin{array}{cc}
X_{1} & Y_{1} \\
Y_{1}^{T} & 0
\end{array}\right]-(1-\alpha)\left[\begin{array}{cc}
0 & Y_{2} \\
Y_{2}^{T} & X_{2}
\end{array}\right] \geq 0
$$

holds for $\alpha=0,1$, then the following inequality

$$
\begin{aligned}
& {\left[\begin{array}{cc}
\frac{1}{\alpha} R_{1} & 0 \\
0 & \frac{1}{1-\alpha} R_{2}
\end{array}\right] \geq\left[\begin{array}{cc}
R_{1} & 0 \\
0 & R_{2}
\end{array}\right]+(1-\alpha)\left[\begin{array}{cc}
X_{1} & Y_{2} \\
Y_{2}^{T} & 0
\end{array}\right]} \\
& +\alpha\left[\begin{array}{cc}
0 & Y_{1} \\
Y_{1}^{T} & X_{2}
\end{array}\right]
\end{aligned}
$$

holds for all $\alpha \in(0,1)$.

Lemma 4 (see [3]). $\mathcal{S}_{1}, \mathcal{S}_{2}$ and $\mathcal{S}_{3}$ are given constant matrices, where $\delta_{1}^{T}=\mathcal{S}_{1}, \mathcal{S}_{2}=\mathcal{S}_{2}^{T}$. If and only if

$$
\begin{array}{r}
{\left[\begin{array}{cc}
\mathcal{S}_{1} & \mathcal{S}_{3} \\
\mathcal{S}_{3}^{T} & -\mathcal{S}_{2}
\end{array}\right]<0} \\
\text { or }\left[\begin{array}{cc}
-\mathcal{S}_{2} & \mathcal{S}_{3} \\
\mathcal{S}_{3}^{T} & \mathcal{S}_{1}
\end{array}\right]<0,
\end{array}
$$

then we have $\mathcal{S}_{1}+\mathcal{S}_{3}^{T} \mathcal{S}_{2}^{-1} \mathcal{S}_{3}<0$.

Lemma 5 (see [42]). For any matrix $R \in \mathbb{S}_{+}^{n}$, integer $N \geq 0$, time functions $a, b, a<b$, and a function $x$ in $\mathscr{L}_{2}([a, b]) \longrightarrow$ $\left.\mathbb{R}^{n}\right)$, the inequality

$$
\int_{a}^{b} x^{T}(s) R x(s) d s \geq(b-a) \phi_{N}^{T} \bar{R}_{N} \phi_{N}
$$

holds, where

$$
\begin{aligned}
\phi_{N} & =\frac{1}{b-a} \int_{a}^{b} \mathbb{L}_{N}\left(\frac{s-a}{b-a}\right) x(s) d s \\
\bar{R}_{N} & =\operatorname{diag}(R, 3 R, \ldots,(2 N+1) R) .
\end{aligned}
$$

Lemma 6 (see [29]). For any given positive matrices $L>0$, $Y>0$, if the following inequality holds

$$
(L-Y) Y^{-1}(L-Y)>0,
$$

then we have

$$
L Y^{-1} L>2 L-Y \text {. }
$$

Remark 7. For the networked control system, we have designed an improved event-triggered generator in this section. Under the nonlinear function and improved eventtriggered condition, the time delay problem will be reconsidered. Based on above preliminaries, we will give the related stability analysis in the next section.

\section{Stability Criteria}

In this section, let us investigate the stability problem of nonlinear networked control system. Compared with the previous networked control system, we will employ the Bessel-Legendre inequality method to reduce the delay upper bound conservatism of the nonlinear networked control system with time delay. At first, the relevant properties of the Legendre polynomials will be introduced. For any given matrix $R \in \mathbb{S}_{+}^{n}$, it holds that

$$
\int_{0}^{1} \mathbb{L}_{N}(\mu) R^{-1} \mathbb{L}_{N}^{T}(\mu) d \mu=\bar{R}_{N}^{-1},
$$

where

$$
\begin{aligned}
\bar{R}_{N} & =\operatorname{diag}\{R, 3 R, \ldots,(2 N+1) R\} \\
\mathbb{E}_{N}(1) & =\left[\begin{array}{c}
I_{n} \\
I_{n} \\
\vdots \\
I_{n}
\end{array}\right]:=1_{N},
\end{aligned}
$$

$$
\mathbb{L}_{N}(0)=\left[\begin{array}{c}
I_{n} \\
-I_{n} \\
\vdots \\
(-1)^{N} I_{n}
\end{array}\right]:=\overline{1}_{N} \text {. }
$$

According to the properties of the orthogonal polynomials, the Legendre polynomials satisfy

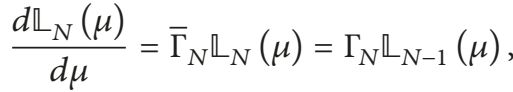

$$
\begin{aligned}
& \frac{d\left(\mu \mathbb{L}_{N}(\mu)\right)}{d \mu}=\mathbb{L}_{N}(\mu)+\Theta_{N} \mathbb{L}_{N}(\mu),
\end{aligned}
$$

where $\bar{\Gamma}_{N}=\left[\begin{array}{ll}\Gamma_{N} & 0_{n(N+1), n}\end{array}\right], \Gamma_{N}=\gamma_{N} \otimes I_{n}$ and $\Theta_{N}=\theta_{N} \otimes I_{n}$, matrices $\gamma_{N} \in \mathbb{R}^{(N+1) \times N}$ and $\theta_{N} \in \mathbb{R}^{(N+1) \times(N+1)}$ are defined as

$$
\begin{aligned}
& \gamma_{N}(k, i)= \begin{cases}0 & \text { if } k \geq i, \\
(2 k-1)\left(1-(-1)^{i+k}\right) & \text { if } k<i,\end{cases} \\
& \theta_{N}(k, i)= \begin{cases}0 & \text { if } k \geq i, \\
k & \text { if } k=i, \\
(2 k-1)\left(1-(-1)^{i+k}\right) & \text { if } k<i .\end{cases}
\end{aligned}
$$

Theorem 8. For given $N \in \mathbb{N}$, the system (14) is stable if there exist any matrices $P_{N} \in \mathbb{S}_{+}^{(2 N+3) n}, Q_{1} \in \mathbb{S}_{+}^{n}, Q_{2} \in \mathbb{S}_{+}^{n}, R \in \mathbb{S}_{+}^{n}$, 
$\Omega \in \mathbb{S}_{+}^{n}$, and $Y_{1} \in \mathbb{R}^{(N+2) n \times(N+2) n}, Y_{2} \in \mathbb{R}^{(N+2) n \times(N+2) n}$ such that the following inequality holds for all $(d(t), \dot{d}(t)) \in \mathscr{H}$ :

$\Xi$

$$
=\left[\begin{array}{ccc}
\Xi_{N 0}(d(t), \dot{d}(t)) & W_{N}^{T} \mathbb{Y}_{1} & W_{N}^{T} \mathbb{Y}_{2} \\
* & -\frac{d_{2}-d(t)}{d_{2}} \widetilde{R}_{N+1} & 0 \\
* & * & -\frac{d(t)}{d_{2}} \widetilde{R}_{N+1}
\end{array}\right]
$$

$\leq 0$,

where

$$
\begin{aligned}
& \mathscr{H}=\mathscr{C} o\left\{(0,0),\left(0, d_{M}\right),\left(d_{2}, 0\right),\left(d_{2}, d_{m}\right)\right\}, \\
& H_{N}=\left[\begin{array}{cccccc}
0 & 0 & 0 & 0 & 0 & 0 \\
0 & \overline{1}_{N} & 0 & 0 & \bar{\Gamma}_{N}-\Theta_{N} & 0 \\
0 & -\overline{1}_{N} & 0 & 0 & 0 & \Theta_{N}
\end{array}\right], \\
& J_{N}=\left[\begin{array}{cccccc}
A+A_{\tau} F & B K & B K & 0 & 0 & 0 \\
1_{N} & -\overline{1}_{N} & 0 & 0 & -\bar{\Gamma}_{N} & 0 \\
0 & 1_{N} & 0 & -\overline{1}_{N} & 0 & -\bar{\Gamma}_{N}
\end{array}\right] \text {, } \\
& W_{N}=\left[\begin{array}{cccccc}
1_{N+1} & -\overline{1}_{N+1} & 0 & 0 & -\Gamma_{N+1} & 0 \\
0 & 1_{N+1} & 0 & -\overline{1}_{N+1} & 0 & -\Gamma_{N+1}
\end{array}\right] \text {, } \\
& \Lambda_{N}=\left[\begin{array}{cc}
\bar{R}_{N+1} & 0 \\
0 & \bar{R}_{N+1}
\end{array}\right]+\frac{d_{2}-d(t)}{d_{2}}\left[\begin{array}{cc}
\bar{R}_{N+1} & Y_{2} \\
Y_{2}^{T} & 0
\end{array}\right] \\
& +\frac{d(t)}{d_{2}}\left[\begin{array}{cc}
0 & Y_{1} \\
Y_{1}^{T} & \bar{R}_{N+1}
\end{array}\right] \\
& \mathbb{Y}_{1}=\left[\begin{array}{c}
\frac{d_{2}-d(t)}{d_{2}} Y_{1} \\
0
\end{array}\right] \text {, } \\
& \mathbb{Y}_{2}=\left[\begin{array}{c}
0 \\
\frac{d(t)}{d_{2}} Y_{2}^{T}
\end{array}\right] \text {, }
\end{aligned}
$$$$
F_{N}=\left[\begin{array}{llllll}
A+A_{\tau} F & B K & B K & 0 & 0 & 0
\end{array}\right] \text {, }
$$$$
\widetilde{R}_{N+1}=\operatorname{diag}(R, 3 R, \ldots,(2 N+1) R,(2 N+3) R),
$$$$
G_{N}(d(t))
$$$$
=\left[\begin{array}{cccccc}
I_{n} & 0 & 0 & 0 & 0 & 0 \\
0 & 0 & 0 & 0 & d(t) I_{n N} & 0 \\
0 & 0 & 0 & 0 & 0 & \left(d_{2}-d(t)\right) I_{n N}
\end{array}\right],
$$$$
\Sigma_{N}(\dot{d}(t))=\operatorname{diag}\left(Q_{1},-(1-\dot{d}(t))\left(Q_{1}-Q_{2}\right)\right.
$$$$
\left.+\sigma_{k}(t) \Omega,-\Omega,-Q_{2}, 0,0\right),
$$

$$
\begin{aligned}
& \Xi_{N 0}(d(t), \dot{d}(t)) \\
& \quad=H e\left(G_{N}^{T}(d(t)) P_{N}\left(J_{N}+\dot{d}(t) H_{N}\right)\right) \\
& \quad+\Sigma_{N}(\dot{d}(t))+d_{2}^{2} F_{N}^{T} R F_{N}-W_{N}^{T} \Lambda_{N} W_{N} .
\end{aligned}
$$

Proof. Now we choose the Lyapunov-Krasovskii functional candidate to investigate nonlinear networked control system as

$$
\begin{aligned}
V(x(t), \dot{x}(t))= & V_{1}(x(t), \dot{x}(t))+V_{2}(x(t), \dot{x}(t)) \\
& +V_{3}(x(t), \dot{x}(t)), \\
V_{1}(x(t), \dot{x}(t))= & \tilde{x}^{T}(t) P_{N} \tilde{x}(t), \\
V_{2}(x(t), \dot{x}(t))= & \int_{t-d(t)}^{t} x^{T}(s) Q_{1} x(s) d s \\
& +\int_{t-d_{2}}^{t-d(t)} x^{T}(s) Q_{2} x(s) d s, \\
V_{3}(x(t), \dot{x}(t))= & d_{2} \int_{t-d_{2}}^{t} \int_{\theta}^{t} \dot{x}^{T}(s) R \dot{x}(s) d s d \theta,
\end{aligned}
$$

where

$\tilde{x}^{T}(t)=\left[\begin{array}{lll}x^{T}(t) d(t) \phi_{1, N}^{T}(t) & \left(d_{2}-d(t)\right) \phi_{2, N}^{T}(t)\end{array}\right]$,

$\phi_{1, N}(t)=\frac{1}{d(t)} \int_{t-d(t)}^{t} \mathbb{L}_{N}\left(\frac{s-t+d(t)}{d(t)}\right) x(s) d s$,

$\phi_{2, N}(t)=\frac{1}{d_{2}-d(t)} \int_{t-d_{2}}^{t-d(t)} \mathbb{L}_{N}\left(\frac{s-t+d_{2}}{d_{2}-d(t)}\right) x(s) d s$.

Taking the derivative of $V(x(t), \dot{x}(t))$, we have $\dot{V}(x(t), \dot{x}(t))$

$$
\begin{aligned}
&= \dot{V}_{1}(x(t), \dot{x}(t))+\dot{V}_{2}(x(t), \dot{x}(t)) \\
&+\dot{V}_{3}(x(t), \dot{x}(t)), \\
& \dot{V}_{1}(x(t), \dot{x}(t))=H e\left(\tilde{x}^{T}(t) P_{N} \dot{\tilde{x}}(t)\right), \\
& \dot{V}_{2}(x(t), \dot{x}(t)) \\
&=x^{T}(t) Q_{1} x(t) \\
& \quad-(1-\dot{d}(t)) x^{T}(t-d(t))\left(Q_{1}-Q_{2}\right) x(t-d(t)) \\
& \quad-x^{T}\left(t-d_{2}\right) Q_{2} x\left(t-d_{2}\right),
\end{aligned}
$$

$\dot{V}_{3}(x(t), \dot{x}(t))$

$=d_{2}^{2} \dot{x}^{T}(t) R \dot{x}(t)-d_{2} \int_{t-d_{2}}^{t} \dot{x}(s) R \dot{x}(s) d s$.

Now, we define the

$\xi^{T}(t)$

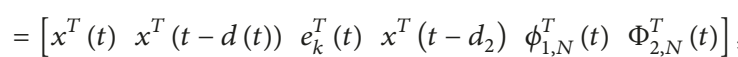


then due to the networked control system model (29), the derivative of $\widetilde{x}(t)$ can be represented by $\dot{\tilde{x}}(\mathrm{t})=\left(J_{N}+\right.$ $\left.\dot{d}(t) H_{N}\right) \xi(t)$, where matrices $J_{N}$ and $H_{N}$ are defined in (31). On the one hand, let us consider

$$
\phi_{N}=\frac{1}{b-a} \int_{a}^{b} \mathbb{L}_{N}\left(\frac{s-a}{b-a}\right) x(s) d s,
$$

where $a$ and $b$ are time functions. Now we set $s(\mu)=(b-a) \mu+$ $a$ to get

$$
\phi_{N}=\int_{0}^{1} \mathbb{L}_{N}(\mu) x(s(\mu)) d \mu
$$

and

$$
\frac{d\left[(b-a) \phi_{N}\right]}{d t}=\dot{a} \psi_{1, N}+(\dot{b}-\dot{a})\left(\psi_{2, N}+\phi_{N}\right),
$$

where

$$
\begin{aligned}
& \psi_{1, N}=(b-a) \int_{0}^{1} \mathbb{L}_{N}(\mu) \dot{x}(s(\mu)) d \mu, \\
& \psi_{2, N}=(b-a) \int_{0}^{1} \mu \mathbb{\complement}_{N}(\mu) \dot{x}(s(\mu)) d \mu .
\end{aligned}
$$

Applying integration by parts, we obtain

$$
\begin{aligned}
& \psi_{1, N}=1_{N} x(b)-\overline{1}_{N} x(a)-\bar{\Gamma}_{N} \phi_{N}, \\
& \psi_{2, N}=1_{N} x(b)-\Theta_{N} \phi_{N}-\phi_{N} .
\end{aligned}
$$

Adding these equations into (39), for all $t \in \mathbb{R}^{+}$,

$$
\begin{aligned}
& \frac{d\left[(b-a) \phi_{N}\right]}{d t} \\
& =\dot{a}\left(1_{N} x(b)-\overline{1}_{N} x(a)-\bar{\Gamma}_{N} \phi_{N}\right) \\
& \left.\quad+(\dot{b}-\dot{a})\left(1_{N} x(b)-\Theta_{N}\right) \phi_{N}-\phi_{N}+\phi_{N}\right) \\
& =\dot{b} 1_{N} x(b)-\dot{a} \overline{1}_{N} x(a)-\left(\dot{a} \bar{\Gamma}_{N}+(\dot{b}-\dot{a}) \Theta_{N}\right) \phi_{N} .
\end{aligned}
$$

Now, let us analyze two cases that $(a, b)=(t-d(t), t)$ and $(a, b)=\left(t-d_{2}, t-d(t)\right)$.

$$
\begin{aligned}
\dot{\tilde{x}} & =\left[\dot{x}^{T}(t) \frac{d\left(d(t) \phi_{1, N}^{T}\right)}{d t} \frac{d\left(d_{2}-d(t)\right) \phi_{2, N}^{T}}{d t}\right], \\
\dot{x}(t) & =A(t)+A_{d} x(t-d(t)),
\end{aligned}
$$

then we have

$$
\begin{aligned}
& \frac{d\left(d(t) \phi_{1, N}\right)}{d t} \\
& =1_{N} x(t)-(1-\dot{d}(t)) \overline{1}_{N} x(t-d(t)) \\
& \quad-\left((1-\dot{d}(t)) \bar{\Gamma}_{N}+\dot{d}(t) \Theta_{N}\right) \phi_{1, N}, \\
& \frac{d\left(\left(d_{2}-d(t)\right) \phi_{2, N}\right)}{d t} \\
& =(1-\dot{d}(t)) 1_{N} x(t-d(t))-\overline{1}_{N} x\left(t-d_{2}\right) \\
& \quad-\left(\bar{\Gamma}_{N}-\dot{d}(t) \Theta_{N}\right) \phi_{2, N} .
\end{aligned}
$$

On the other hand, for the function of $V_{3}(x(t), \dot{x}(t))$, we have

$$
\begin{aligned}
& \int_{t-d_{2}}^{t} \dot{x}^{T}(s) R \dot{x}(s) d s \geq\left[\begin{array}{l}
\widetilde{\phi}_{1, N+1} \\
\widetilde{\phi}_{2, N+1}
\end{array}\right]^{T} \\
& \cdot\left[\begin{array}{cc}
d(t) \widetilde{R}_{N+1} & 0 \\
0 & \left(d_{2}-d(t)\right) \widetilde{R}_{N+1}
\end{array}\right]\left[\begin{array}{l}
\widetilde{\phi}_{1, N+1} \\
\widetilde{\phi}_{2, N+1}
\end{array}\right],
\end{aligned}
$$

where

$$
\begin{aligned}
& \widetilde{\phi}_{1, N+1}=\frac{1}{d(t)} \int_{t-d(t)}^{t} \mathbb{L}_{N+1}\left(\frac{s-t+d(t)}{d(t)}\right) \dot{x}(s) d s, \\
& \widetilde{\phi}_{2, N+1}=\frac{1}{d_{2}-d(t)} \int_{t-d_{2}}^{t-d(t)} \mathbb{L}\left(\frac{s-t+d_{2}}{d_{2}-d(t)}\right) \dot{x}(s) d s .
\end{aligned}
$$

Due to equation (27), we obtain

$$
\begin{aligned}
& {\left[\begin{array}{c}
d(t) \widetilde{\phi}_{1, N+1} \\
\left(d_{2}-d(t)\right) \phi_{2, N+1}
\end{array}\right]} \\
& =\left[\begin{array}{c}
1_{N+1} x(t)-\overline{1}_{N+1} x(t-d(t))-\Gamma_{N+1} \phi_{1, N} \\
1_{N+1} x(t-d(t))-\overline{1}_{N+1} x(t-d(t))-\Gamma_{N+1} \phi_{2, N}
\end{array}\right] \\
& =W_{N} \xi_{N},
\end{aligned}
$$

where $W_{N} \xi_{N}$ is defined in (31).

According to Lemma 5 and Bessel-Legendre inequality, we have

$$
\begin{aligned}
& d_{2} \int_{t-d_{2}}^{t} \dot{x}^{T}(s) R \dot{x}(s) d s \\
& \geq \xi^{T}(t) W_{N}^{T}\left[\begin{array}{cc}
\frac{d_{2}}{d(t)} \widetilde{R}_{N+1} & 0 \\
0 & \frac{d_{2}}{d_{2}-d(t)} \widetilde{R}_{N+1}
\end{array}\right] W_{N} \xi(t) .
\end{aligned}
$$

In addition, for any $Y_{1}, Y_{2} \in \mathbb{R}^{(N+2) n \times(N+2) n}$, consider the matrix

$$
\Lambda_{N 0}=\left[\begin{array}{cc}
\frac{d_{2}-d(t)}{d_{2}} Y_{1} \widetilde{R}_{N+1}^{-1} Y_{1}^{T} & 0 \\
0 & \frac{d(t)}{d_{2}} Y_{2}^{T} \widetilde{R}_{N+1}^{-1} Y_{2}
\end{array}\right]
$$

By applying Lemma 3, we have

$$
\begin{aligned}
& d_{2} \int_{t-d_{2}}^{t} \dot{x}^{T}(s) R \dot{x}(s) d s \\
& \quad \geq \xi^{T}(t) W_{N}^{T}\left[\Lambda_{N}-\Lambda_{N 0}\right] W_{N} \xi(t),
\end{aligned}
$$

where $\Lambda_{N}$ is defined in (31).

In addition, let us consider the following event-triggered condition of the networked control system

$$
e_{k}^{T}(t) \Omega e_{k}(t) \leq \sigma_{k}(t) x^{T}(t-d(t)) \Omega x(t-d(t)) .
$$


Let us add (51) into the derivative of $V(x(t), \dot{x}(t))$, then, we have

$$
\begin{aligned}
\dot{V}(x(t), \dot{x}(t)) & \\
= & H e\left(\xi^{T}(t) G_{N}^{T}(d(t)) P_{N} \dot{\tilde{x}}(t)\right) \\
& +\xi^{T}(t)\left[d_{2}^{2} F_{N}^{T} R F_{N}+\Sigma_{N}(\dot{d}(t))\right] \xi(t) \\
& \quad-d_{2} \int_{t-d_{2}}^{t} \dot{x}^{T}(s) R \dot{x}(s) d s,
\end{aligned}
$$

where $G_{N}, F_{N}, \Sigma_{N}(\dot{d}(t))$ are given in (31).

By the integral inequality method (50), the derivative of Lyapunov-Krasovskii can be rewritten as

$$
\begin{aligned}
\dot{V}( & x(t), \dot{x}(t)) \leq \xi^{T}(t) \\
\cdot & {\left[H e\left(G_{N}^{T}(d(t)) P_{N}\left(J_{N}+\dot{d}(t) H_{N}\right)\right)\right.} \\
+ & \Sigma_{N}(\dot{d}(t))+d_{2}^{2} F_{N}^{T} R F_{N} \\
- & \left.W_{N}^{T}\left[\Xi_{N}(d(t))-\Xi_{N 0}(d(t))\right] W_{N}\right] \xi(t)=\xi^{T}(t) \\
\cdot & \left(\Psi_{N 0}(d(t), \dot{d}(t))+W_{N}^{T} \Xi_{N 0}(d(t)) W_{N}\right) \xi(t) .
\end{aligned}
$$

Notice that the $\Psi_{N 0}(d(t), \dot{d}(t))+W_{N}^{T} \Xi_{N 0}(d(t)) W_{N}$ is multi-affine about $d(t)$ and $\dot{d}(t)$, where $(d(t), \dot{d}(t)) \in \mathscr{H}$. Therefore, by the Schur's complement, if the matrix $\Xi<0$, then $\dot{V}(x(t), \dot{x}(t))<0$ for $(d(t), \dot{d}(t)) \in \mathscr{H}$, the system is stable. This proof is completed.

$$
\Xi=\left[\begin{array}{cc}
\widetilde{\Xi}_{N 0}(d(t), \dot{d}(t)) & W_{N}^{T} \Psi_{1} \\
* & -\frac{d_{2}-d(t)}{d_{2}} \widetilde{R}_{N+1} \\
* & * \\
* & * \\
* & *
\end{array}\right.
$$

holds for all $(d(t), \dot{d}(t)) \in \mathscr{H}$, where

$$
\begin{aligned}
& \mathscr{H}=\mathscr{C} o\left\{(0,0),\left(0, d_{M}\right),\left(d_{2}, 0\right),\left(d_{2}, d_{m}\right)\right\},
\end{aligned}
$$

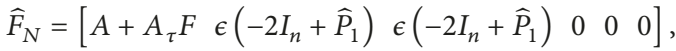

$$
\begin{aligned}
& W_{N}=\left[\begin{array}{cccccc}
1_{N+1} & -\overline{1}_{N+1} & 0 & 0 & -\Gamma_{N+1} & 0 \\
0 & 1_{N+1} & 0 & -\overline{1}_{N+1} & 0 & -\Gamma_{N+1}
\end{array}\right] \text {, } \\
& \Lambda_{N}=\left[\begin{array}{cc}
\bar{R}_{N+1} & 0 \\
0 & \bar{R}_{N+1}
\end{array}\right]+\frac{d_{2}-d(t)}{d_{2}}\left[\begin{array}{cc}
\bar{R}_{N+1} & Y_{2} \\
Y_{2}^{T} & 0
\end{array}\right]+\frac{d(t)}{d_{2}}
\end{aligned}
$$

Remark 9. Notice that LMI (29) is considered satisfying $(d(t), \dot{d}(t)) \in \mathscr{H}$, where

$$
\mathscr{H}=\mathscr{C} o\left\{(0,0),\left(0, d_{M}\right),\left(d_{2}, 0\right),\left(d_{2}, d_{m}\right)\right\} .
$$

That is because the vertices $\left(0, d_{m}\right),\left(d_{2}, d_{M}\right)$ are impossible to reach. In another words, at the lower bound of time delay 0 , the derivative of time delay $\dot{d}(t)$ can not be negative; at the upper bound of time delay $d_{2}$, the derivative of time delay $\dot{d}(t)$ should be non-positive. Thus, we choose the allowable delay set as $\mathscr{H}$.

Remark 10. In this section, the stability problem of the nonlinear networked control system has been discussed. We employed the Bessel-Legendre inequality method to improve the Lyapunov-Krasovskii functional and obtained the delay upper bound. For the $V_{1}(x(t), \dot{x}(t))$, we take the terms $\phi_{1, N}(t)$ and $\phi_{2, N}(t)$ into $\widetilde{x}(t)$ to consider the delay dependent condition. Furthermore, the $V_{2}(x(t), \dot{x}(t))$ will be zoomed by this integral method.

\section{Stabilization of Networked Control System}

In this section, we will deal with the stabilization problem of the event-triggered nonlinear networked control system with time delay. In order to more effectively control the system state and achieve a stable, fast and accurate networked control system, the state feedback controller will be designed. Next, the relevant stabilization theorem is given as follows.

Theorem 11. For given integer scalar $N \geq 0$, scalar $\epsilon>0$, system (14) with the feedback controller gain $K=-\epsilon B^{-1} \widehat{P}_{1}^{-1}$ is stable if there exist matrices $\widehat{P}_{1} \in \mathbb{S}_{+}^{n}, \widehat{P}_{2} \in \mathbb{S}_{+}^{(N+1) n}, \widehat{P}_{3} \in \mathbb{S}_{+}^{(N+1) n}$, $Q_{1}, Q_{2}, R \in \mathbb{S}_{+}^{n}, \Omega \in \mathbb{S}_{+}^{n}$, and $Y_{1}, Y_{2} \in \mathbb{R}^{(N+2) n \times(N+2) n}$, such that

$$
\left.\begin{array}{ccc}
W_{N}^{T} \bigvee_{2} & d_{2}^{2} \widehat{F}_{N}^{T} & -W_{N}^{T} \Lambda_{N} \\
0 & 0 & 0 \\
-\frac{d(t)}{d_{2}} \widetilde{R}_{N+1} & 0 & 0 \\
* & -2 I_{n}+R & 0 \\
* & * & \Lambda_{N}
\end{array}\right] \leq 0
$$

$$
\begin{aligned}
& \cdot\left[\begin{array}{cc}
0 & Y_{1} \\
Y_{1}^{T} & \bar{R}_{N+1}
\end{array}\right] \\
& \widetilde{R}_{N+1}=\operatorname{diag}(R, 3 R, \ldots,(2 N+1) R,(2 N+3) R), \\
& G_{N}(d(t))=\left[\begin{array}{cccccc}
I_{n} & 0 & 0 & 0 & 0 & 0 \\
0 & 0 & 0 & 0 & d(t) I_{n N} & 0 \\
0 & 0 & 0 & 0 & 0 & \left(d_{2}-d(t)\right) I_{n N}
\end{array}\right], \\
& \Sigma_{N}(\dot{d}(t))=\operatorname{diag}\left(Q_{1},-(1-\dot{d}(t))\left(Q_{1}-Q_{2}\right)+\sigma_{k}(t) \Omega\right. \text {, } \\
& \left.-\Omega,-Q_{2}, 0,0\right) \text {, }
\end{aligned}
$$




$$
\begin{aligned}
& \widetilde{\Xi}_{N 0}(d(t), \dot{d}(t))=H e\left(G_{N}^{T}(d(t)) \bar{\Xi}_{N 0}(\dot{d}(t))\right)+\Sigma_{N}(\dot{d}(t)), \\
& \bar{\Xi}_{N 0}(\dot{d}(t)) \\
& =\left[\begin{array}{cccccc}
\widehat{P}_{1}\left(A+A_{\tau} F\right) & -\epsilon I_{n} & -\epsilon I_{n} & 0 & 0 & 0 \\
\widehat{P}_{2}\left(1_{N}\right) & -\widehat{P}_{2} \overline{1}_{N} & 0 & 0 & -\widehat{P}_{2} \bar{\Gamma}_{N} & 0 \\
0 & \widehat{P}_{3} 1_{N} & 0 & -\widehat{P}_{3} \overline{1}_{N} & 0 & -\widehat{P}_{3} \bar{\Gamma}_{N}
\end{array}\right] \\
& +\dot{d}(t)\left[\begin{array}{cccccc}
0 & 0 & 0 & 0 & 0 & 0 \\
0 & \widehat{P}_{2} \overline{1}_{N} & 0 & 0 & \widehat{P}_{2} \bar{\Gamma}_{N}-\widehat{P}_{2} \Theta_{N} & 0 \\
0 & -\widehat{P}_{3} \overline{1}_{N} & 0 & 0 & 0 & \widehat{P}_{3} \Theta_{N}
\end{array}\right] \text {, } \\
& \mathbb{Y}_{1}=\left[\begin{array}{c}
\frac{d_{2}-d(t)}{d_{2}} Y_{1} \\
0
\end{array}\right] \text {, } \\
& \mathbb{Y}_{2}=\left[\begin{array}{c}
0 \\
\frac{d(t)}{d_{2}} Y_{2}^{T}
\end{array}\right] \text {, }
\end{aligned}
$$

Proof. According to Theorem 8 and Lemma 4, we have obtained the LMI as follows:

$$
=\left[\begin{array}{cccc}
\widehat{\Xi}_{N 0}(d(t), \dot{d}(t)) & W_{N}^{T} \mathbb{Y}_{1} & W_{N}^{T} \mathbb{Y}_{2} & d_{2}^{2} F_{N}^{T} \\
* & -\frac{d_{2}-d(t)}{d_{2}} \widetilde{R}_{N+1} & 0 & 0 \\
* & * & -\frac{d(t)}{d_{2}} \widetilde{R}_{N+1} & 0 \\
* & * & * & -R^{-1}
\end{array}\right]
$$

$\leq 0$,

where $\widehat{\Xi}_{N 0}(d(t), \dot{d}(t))=H e\left(G_{N}^{T} P_{N}\left(J_{N}+\dot{d}(t) H_{N}\right)\right)+\Sigma_{N}(\dot{d}(t))+$ $W_{N}^{T} \Lambda_{N} W_{N}$.

For the symmetric matrix $P_{N}$, we set

$$
P_{N}=\left[\begin{array}{ccc}
\widehat{P}_{1} & 0 & 0 \\
0 & \widehat{P}_{2} & 0 \\
0 & 0 & \widehat{P}_{3}
\end{array}\right],
$$

where $\widehat{P}_{1} \in \mathbb{S}_{+}^{n}, \widehat{P}_{2} \in \mathbb{S}_{+}^{(N+1) n}$ and $\widehat{P}_{3} \in \mathbb{S}_{+}^{(N+1) n}$. Then we have

$$
G_{N}^{T} \bar{\Xi}_{N 0}(d(t), \dot{d}(t))=G_{N}^{T} P_{N}\left(J_{N}+\dot{d}(t) H_{N}\right)
$$

$$
\begin{aligned}
& =G_{N}^{T}\left[\begin{array}{cccc}
\hat{P}_{1} & 0 & 0 \\
0 & \widehat{P}_{2} & 0 \\
0 & 0 & \widehat{P}_{3}
\end{array}\right]\left(\left[\begin{array}{cccccc}
A+A_{\tau} F & B K & B K & 0 & 0 & 0 \\
1_{N} & -\overline{1}_{N} & 0 & 0 & -\bar{\Gamma}_{N} & 0 \\
0 & 1_{N} & 0 & -\overline{1}_{N} & 0 & -\bar{\Gamma}_{N}
\end{array}\right]+\dot{d}(t)\right. \\
& \left.\cdot\left[\begin{array}{cccccc}
0 & 0 & 0 & 0 & 0 & 0 \\
0 & \overline{1}_{N} & 0 & 0 & \bar{\Gamma}_{N}-\Theta_{N} & 0 \\
0 & -\overline{1}_{N} & 0 & 0 & 0 & \Theta_{N}
\end{array}\right]\right) .
\end{aligned}
$$

At the same time, we set the controller gain $K=-\epsilon B^{-1} \widehat{P}_{1}^{-1}$, then we obtain

$$
\begin{aligned}
& G_{N}^{T} \bar{\Xi}_{N 0}(d(t), \dot{d}(t)) \\
& \quad=G_{N}^{T}\left(\left[\begin{array}{cccccc}
\widehat{P}_{1}\left(A+A_{\tau} F\right) & -\epsilon I_{n} & -\epsilon I_{n} & 0 & 0 & 0 \\
\widehat{P}_{2}\left(1_{N}\right) & -\widehat{P}_{2} \overline{1}_{N} & 0 & 0 & -\widehat{P}_{2} \bar{\Gamma}_{N} & 0 \\
0 & \widehat{P}_{3} 1_{N} & 0 & -\widehat{P}_{3} \overline{1}_{N} & 0 & -\widehat{P}_{3} \bar{\Gamma}_{N}
\end{array}\right]+\dot{d}(t)\left[\begin{array}{cccccc}
0 & 0 & 0 & 0 & 0 & 0 \\
0 & \widehat{P}_{2} \overline{1}_{N} & 0 & 0 & \widehat{P}_{2} \bar{\Gamma}_{N}-\widehat{P}_{2} \Theta_{N} & 0 \\
0 & -\widehat{P}_{3} \overline{1}_{N} & 0 & 0 & 0 & \widehat{P}_{3} \Theta_{N}
\end{array}\right]\right) .
\end{aligned}
$$

Thanks to Lemma 6, we replace $-R^{-1}$ with $-2 I_{n}+R$; on the other hand, define

$$
F_{N}=\left[A+A_{\tau} F \quad \epsilon\left(-2 I_{n}+\widehat{P}_{1}\right) \quad 0 \quad 0 \quad 0\right] .
$$

Combining equations (58), (59), (60) and (61), we obtain the following inequality:

$$
\left[\begin{array}{cccc}
\widehat{\Xi}_{N 0}(d(t), \dot{d}(t)) & W_{N}^{T} \bigvee_{1} & W_{N}^{T} \bigvee_{2} & d_{2}^{2} F_{N}^{T} \\
* & -\frac{d_{2}-d(t)}{d_{2}} \widetilde{R}_{N+1} & 0 & 0 \\
* & * & -\frac{d(t)}{d_{2}} \widetilde{R}_{N+1} & 0 \\
* & * & * & -2 I_{n}+R
\end{array}\right]
$$

In addition, apply the Schur's complement to (62) again. Then, linear matrix inequality is obtained in Theorem 11 . This proof is completed.

\section{Numerical Examples}

Example 1. In order to understand the applicability of the system more clearly, and demonstrate the effectiveness of Bessel-Legendre inequalities method, we give Example 1. First of all, let us consider the system model as follows:

$$
\dot{x}(t)=A x(t)+A_{\tau} F x(t)+B u(t),
$$

and the system relevant parameters are given as

$$
A=\left[\begin{array}{cc}
-2 & 0 \\
0 & -0.9
\end{array}\right] \text {, }
$$


TABLE 1: Delay upper bound $d_{2}$ for different $\dot{d}(t)\left(d_{M}=-d_{m}=\dot{d}(t)\right)$.

\begin{tabular}{lcccc}
\hline Method $\backslash \dot{d}(t)$ & 0.1 & 0.3 & 0.5 & 0.9 \\
\hline Theorem $8, \mathrm{~N}=3$ & 4.7526 & 3.01780 & 3.0472 & 2.9856 \\
\hline Theorem $8, \mathrm{~N}=2$ & 3.2546 & 2.3136 & 2.1797 & 2.0732 \\
\hline Theorem $8, \mathrm{~N}=1$ & 2.8572 & 2.6095 & 1.0755 & 2.0153 \\
\hline Theorem $8, \mathrm{~N}=0$ & 2.3544 & 1.1498 & 0.8671 & 0.0526 \\
\hline Theorem 1 of [45] & 2.1403 & 1.0653 & 0.6496 & 0.7644 \\
\hline Theorem 1 of [46] & 0.7522 & 0.7197 & 0.9775 \\
\hline Theorem 1 of [13] & 1.2890 & 1.0126 & 0.6324 \\
\hline
\end{tabular}

$$
\begin{aligned}
A_{\tau} & =\left[\begin{array}{cc}
-1 & 0 \\
-1 & -1
\end{array}\right], \\
B & =\left[\begin{array}{cc}
-1 & 0 \\
-0.5 & -1
\end{array}\right], \\
F & =\left[\begin{array}{cc}
-1 & 0 \\
-0.5 & -1
\end{array}\right] .
\end{aligned}
$$

For testifying the less conservatism of the obtained stability condition in this paper, the comparison with other paper's results about the upper bound of delay is given in Table 1. From Table 1 , for $N=0, \dot{d}(t)$ is taken different values as $0.1,0.3,0.5,0.9$, respectively, then the obtained upper bound of time delays are 2.3544, 1.1498, 1.0755, 1.0526, respectively, which are all larger than the values in $[13,45$, $46]$ for the corresponding values of $\dot{d}(t)$. Obviously, for this nonlinear networked control system, the conservatism of the stability criteria derived by using Bessel-Legendre inequality method in this paper has been greatly reduced. Furthermore, the obtained stability criteria are related to the order $N$. As we can see in Table 1, the upper bound of time delay increases with $N$ increasing. In other words, the larger $N$, the lower conservatism.

Next, we propose Example 2 to investigate the triggering performance of nonlinear networked control system under the improved event-triggered scheme.

Example 2. This example concerns the parameters of nonlinear networked control system as follows:

$$
\begin{aligned}
A & =\left[\begin{array}{cccc}
0 & 0.5 & 0 & 0 \\
0 & 0 & -3 & 0 \\
-1 & 0 & 0 & 1 \\
0.5 & 0 & -3 & 0.7
\end{array}\right], \\
A_{\tau} & =\left[\begin{array}{cccc}
0 & 0.1 & 0 & 0 \\
0 & 0 & 1 & 0 \\
-1 & 0 & 0 & 1 \\
0.5 & 0 & -3 & 1
\end{array}\right],
\end{aligned}
$$

$$
\begin{aligned}
& B=\left[\begin{array}{c}
0 \\
0.1 \\
0 \\
-0.01
\end{array}\right], \\
& F=\left[\begin{array}{cccc}
5 & 0 & 0 & 0 \\
0 & -0.3 & 0 & 0 \\
0 & 2.0 & -0.6667 & 0.6667 \\
0 & -2.7 & 0.1 & -0.8
\end{array}\right] .
\end{aligned}
$$

Set $\sigma_{k 1}=0.01, \bar{\sigma}=0.6, h=0.22, \dot{d}(t)=0.1$. Under the improved event-triggered scheme, the obtained controller gains $K$ and triggering parameters $\Omega$ at $t=0, t=1, \ldots, t=$ 30 are shown in Table 2.

In this improved event-triggered scheme, the parameter $\sigma_{k}(t)$ is time-varying and satisfies (10), which can change the release rate at different times while saving the network transmission resource. When $h=0.22, \lambda=0.01$, the variation of $\sigma_{k}(t)$ and release instants are shown in Figure 2. In Figure 2, there are only 29 sampled signals which takes $21.3 \%$ of the whole sampled signals need to be sent out when $t \in[0,30]$. In addition, from Figure 2 , we can see that the parameter $\sigma_{k}(t)$ varies from 0.01 to 0.4 and the frequency of triggering in initial times is higher than other times, which can shorten the dynamic process.

To further illustrate the advantage of our improved static event-triggered scheme, we compare it with the general static event-triggered scheme in which the parameter $\sigma_{k}(t)$ is time invariant. We set $h=0.12$, then the results are shown in Figures 3 and 4 . Obviously, the release times in initial times in Figure 4 are more than those in Figure 3 and there are more packets that can be transmitted in initial times.

The state response of the event-triggered nonlinear networked control system is shown in Figure 5. At 3 seconds, the system achieves steady.

\section{Conclusions}

In this paper, we discussed the stability and stabilization problem of the event-triggered nonlinear networked control 
TABLE 2: The controller gains $K$ and triggering parameters $\Omega$ at different times.

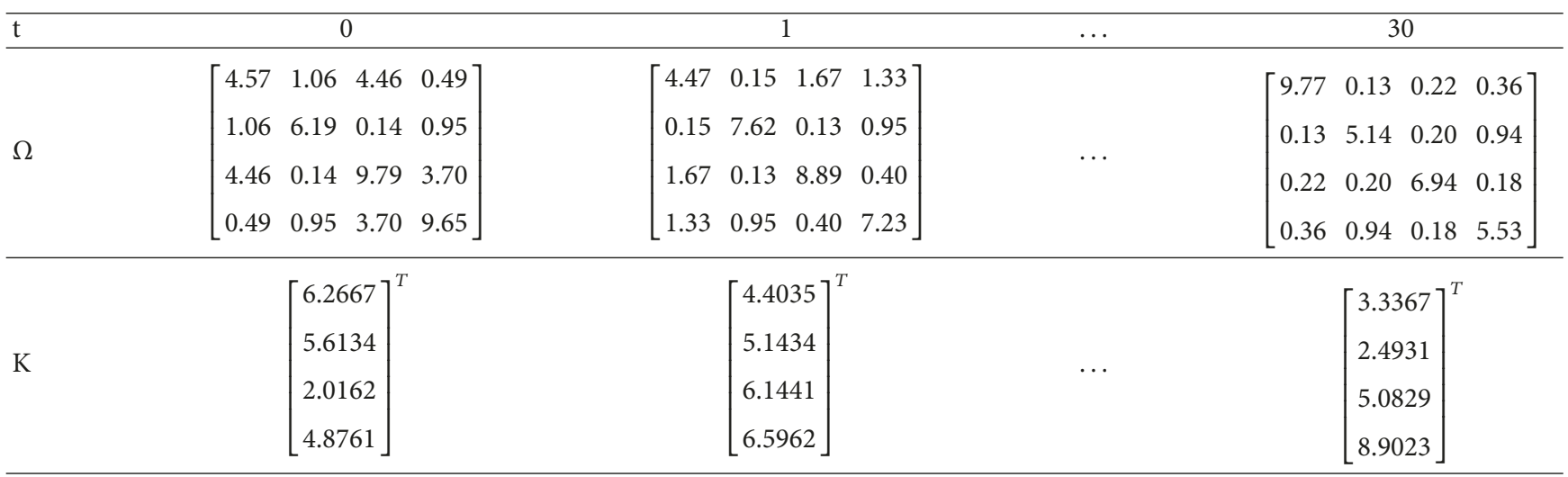
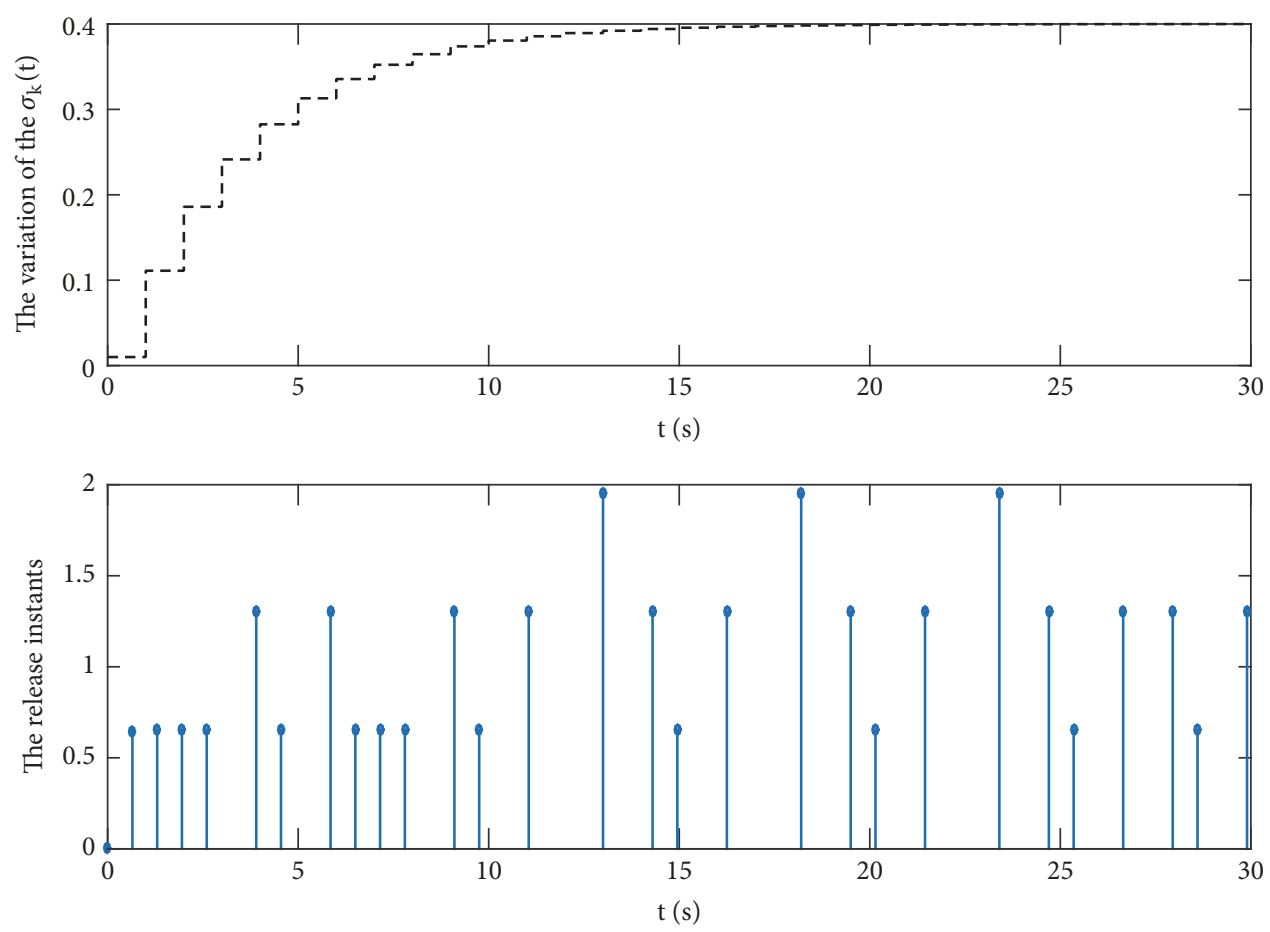

FIgURE 2: The variation of $\sigma_{k}(t)$ and release instants, $\mathrm{h}=0.22$.

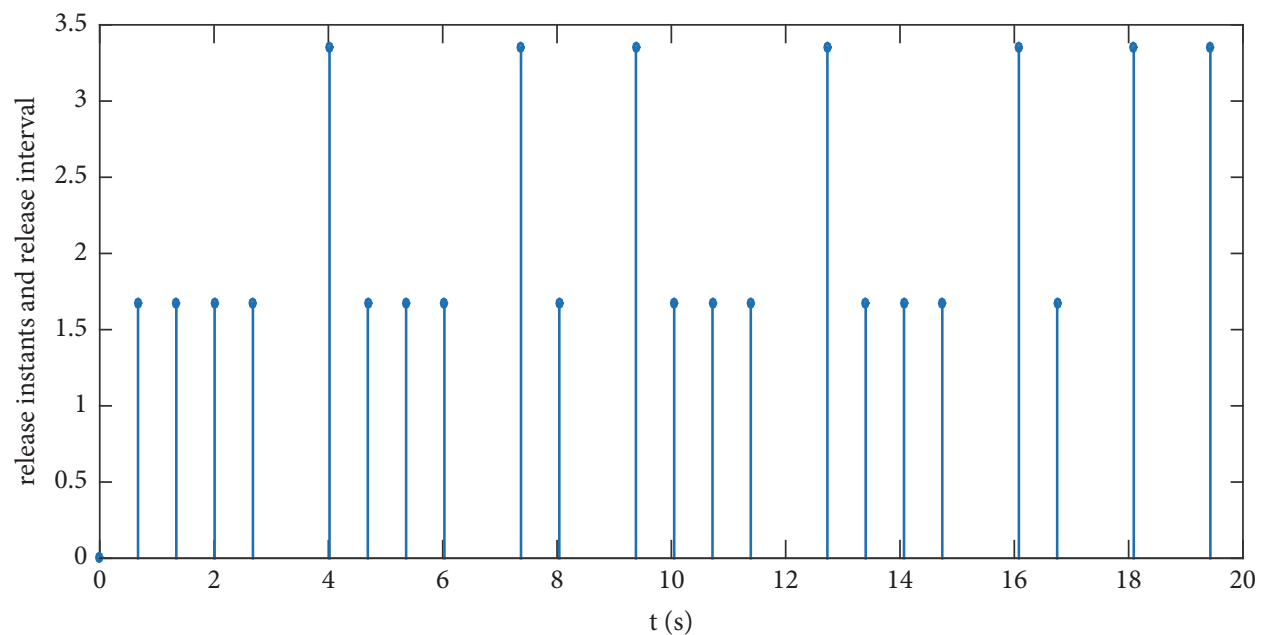

FIGURE 3: Improved static event-based release interval and release instants, $\mathrm{h}=0.12$. 


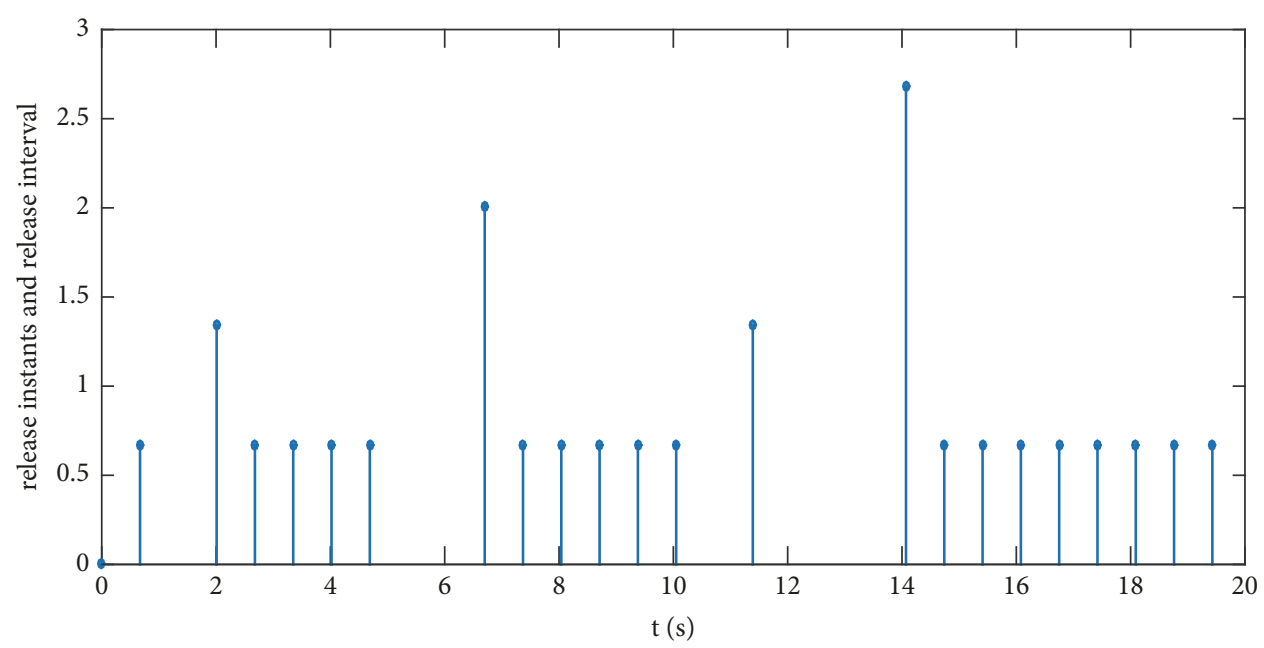

FIGURE 4: Static event-based release interval and release instants, $\mathrm{h}=0.12$.

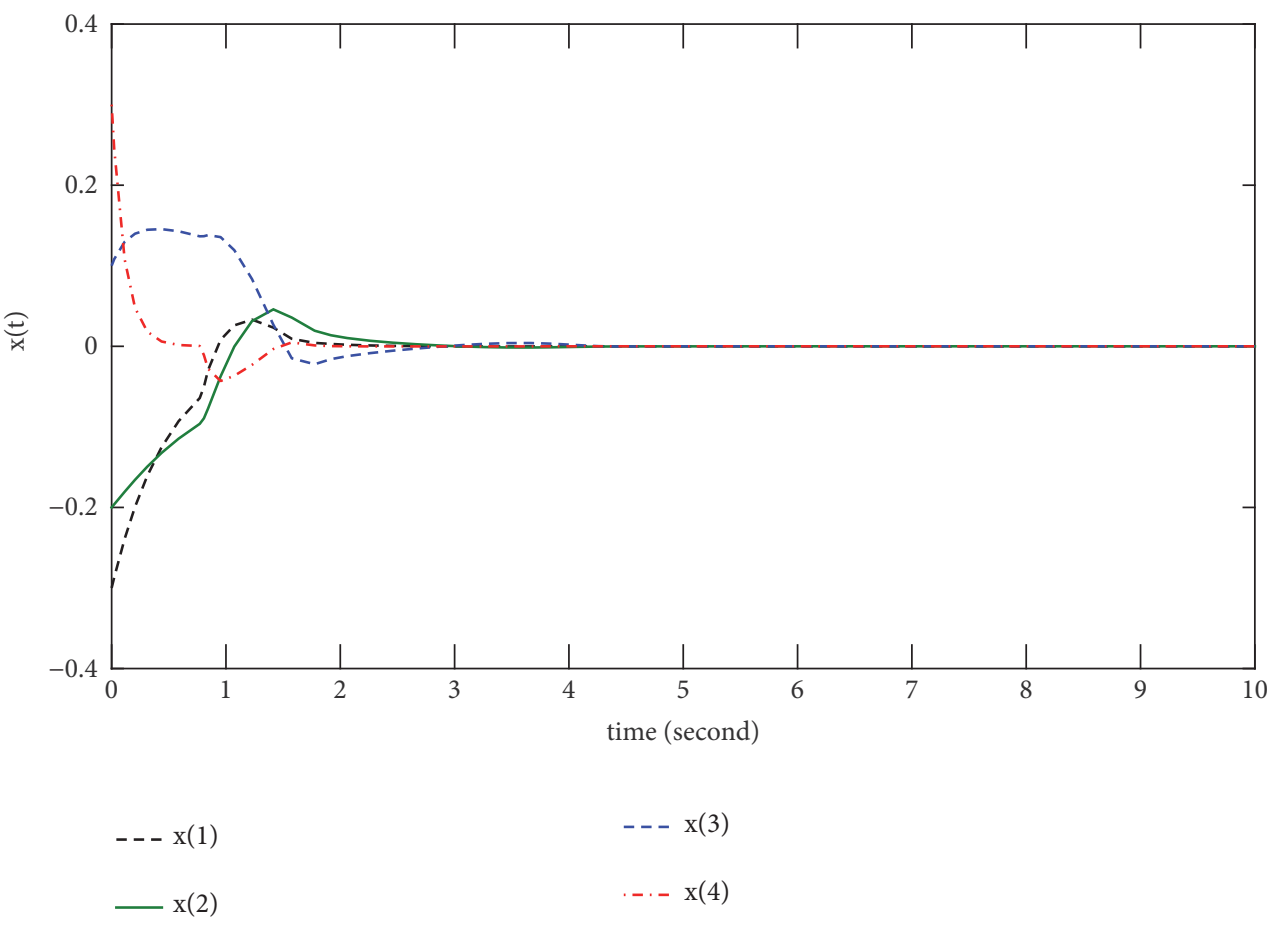

FIGURE 5: State response of the event-trigger nonlinear networked control system.

system with time varying delay. Firstly, on one hand, compared with the previous investigation of networked control system, this paper utilizes the Bessel-Legendre inequality method to reduce the conservatism of the system delay upper bound. On the other hand, by designing an appropriate state feedback controller, the stabilization problem of the nonlinear networked control system has been solved. Secondly, an improved ETS was put forward to reduce transmission load and make the system has a better dynamic process. Finally, two simulation examples have been shown to verify the effectiveness of the improved time delay method and ETS. Further study can be concentrated on the discrete time matters for the networked control system and finite time issues for nonlinear networked control switch system under an improved ETS.

\section{Data Availability}

The data used to support the findings of this study are included within the article.

\section{Conflicts of Interest}

The authors declare that they have no conflicts of interest. 


\section{Acknowledgments}

This work is supported by the Natural Science Foundation of Shandong Province under Grant No. ZR2014FM019, National Natural Science Foundation of China under Grant Nos. 61573095, and Shandong Province Higher Educational Science and Technology Program No. J15LN04.

\section{References}

[1] H. Gao, T. Chen, and J. Lam, "A new delay system approach to network-based control," Automatica, vol. 44, no. 1, pp. 39-52, 2008.

[2] G. Chen, J. Xia, G. Zhuang, and J. Zhao, "Improved delaydependent stabilization for a class of networked control systems with nonlinear perturbations and two delay components," Applied Mathematics and Computation, vol. 316, pp. 1-17, 2018.

[3] L. Ma, Z. Wang, H.-K. Lam, and N. Kyriakoulis, "Distributed event-based set-membership filtering for a class of nonlinear systems with sensor saturations over sensor networks," IEEE Transactions on Cybernetics, vol. 47, no. 11, pp. 3772-3783, 2016.

[4] H. Kim and N. Feamster, "Improving network management with software defined networking," IEEE Communications Magazine, vol. 51, no. 2, pp. 114-119, 2013.

[5] E. Tian and D. Yue, "Decentralized control of network-based interconnected systems: a state-dependent triggering method," International Journal of Robust and Nonlinear Control, vol. 25, no. 8, pp. 1126-1144, 2015.

[6] X. Wang and M. D. Lemmon, "Event-triggering in distributed networked control systems," Institute of Electrical and Electronics Engineers Transactions on Automatic Control, vol. 56, no. 3, pp. 586-601, 2011.

[7] H. Zhang, P. Cheng, L. Shi, and J. Chen, "Optimal DoS attack scheduling in wireless networked control system," IEEE Transactions on Control Systems Technology, vol. 24, no. 3, pp. 843-852, 2015.

[8] G. C. Walsh, O. Beldiman, and L. G. Bushnell, "Asymptotic behavior of nonlinear networked control systems," Institute of Electrical and Electronics Engineers Transactions on Automatic Control, vol. 46, no. 7, pp. 1093-1097, 2001.

[9] B. Tavassoli, "Stability of nonlinear networked control systems over multiple communication links with asynchronous sampling," IEEE Transactions on Automatic Control, vol. 59, no. 2, pp. 511-515, 2014.

[10] G. Wang, L. Li, and B. Wu, "Robust stability of nonlinear model-based networked control systems with time-varying transmission times," Nonlinear Dynamics, vol. 69, no. 3, pp. 1351-1363, 2012.

[11] L. Su and G. Chesi, "Robust stability analysis and synthesis for uncertain discrete-time networked control systems over fading channels," Institute of Electrical and Electronics Engineers Transactions on Automatic Control, vol. 62, no. 4, pp. 1966-1971, 2017.

[12] H.-B. Zeng, J. H. Park, S.-P. Xiao, and Y. Liu, "Further results on sampled-data control for master-slave synchronization of chaotic Lur'e systems with time delay," Nonlinear Dynamics, vol. 82, no. 1-2, pp. 851-863, 2015.

[13] H.-B. Zeng, Y. He, M. Wu, and S.-P. Xiao, "Absolute stability and stabilization for Lurie networked control systems," International Journal of Robust and Nonlinear Control, vol. 21, no. 14, pp. 16671676, 2011.
[14] D. E. Quevedo, V. Gupta, W.-J. Ma, and S. Yüksel, "Stochastic stability of event-triggered anytime control," IEEE Transactions on Automatic Control, vol. 59, no. 12, pp. 3373-3379, 2013.

[15] H. Li, Z. Chen, L. Wu, H. Lam, and H. Du, "Event-triggered fault detection of nonlinear networked systems," IEEE Transactions on Cybernetics, vol. 47, no. 4, pp. 1041-1052, 2017.

[16] E. Garcia, Y. Cao, and D. W. Casbeer, "Periodic event-triggered synchronization of linear multi-agent systems with communication delays," IEEE Transactions on Automatic Control, vol. 62, no. 1, pp. 366-371, 2017.

[17] D. Yang, W. Ren, X. Liu, and W. Chen, "Decentralized eventtriggered consensus for linear multi-agent systems under general directed graphs," Automatica, vol. 69, pp. 242-249, 2016.

[18] E. Tian, Z. Wang, L. Zou, and D. Yue, "Probabilistic-constrained filtering for a class of nonlinear systems with improved static event-triggered communication," International Journal of Robust and Nonlinear Control, vol. 29, no. 5, pp. 1484-1498, 2019.

[19] X. Ge, Q. Han, and Z. Wang, "A dynamic event-triggered transmission scheme for distributed set-membership estimation over wireless sensor networks," IEEE Transactions on Cybernetics, vol. 99, pp. 1-13, 2019.

[20] A. Seuret, F. Gouaisbaut, and Y. Ariba, "Complete quadratic Lyapunov functionals for distributed delay systems," Automatica, vol. 62, pp. 168-176, 2015.

[21] X.-M. Zhang, Q.-L. Han, and Z. Zeng, "Hierarchical type stability criteria for delayed neural networks via canonical bessel-legendre inequalities," IEEE Transactions on Cybernetics, vol. 48, no. 5, pp. 1660-1671, 2018.

[22] B. Liu and X.-C. Jia, "New absolute stability criteria for uncertain Lure systems with time-varying delays," Journal of The Franklin Institute, vol. 355, no. 9, pp. 4015-4031, 2018.

[23] H. H. Choi and M. J. Chung, "Observer-based Ho controller design for state delayed linear systems," Automatica, vol. 32, no. 7, pp. 1073-1075, 1996.

[24] A. Seuret and F. Gouaisbaut, "Hierarchy of LMI conditions for the stability analysis of time-delay systems," Systems \& Control Letters, vol. 81, pp. 1-7, 2015.

[25] O. Solomon and E. Fridman, "New stability conditions for systems with distributed delays," Automatica, vol. 49, no. 11, pp. 3467-3475, 2013.

[26] W.-H. Chen and W. X. Zheng, "Delay-dependent robust stabilization for uncertain neutral systems with distributed delays," Automatica, vol. 43, no. 1, pp. 95-104, 2007.

[27] P. G. Park, J. W. Ko, and C. Jeong, "Reciprocally convex approach to stability of systems with time-varying delays," Automatica, vol. 47, no. 1, pp. 235-238, 2011.

[28] W.-J. Lin, Y. He, C.-K. Zhang, and M. Wu, "Stability analysis of neural networks with time-varying delay: Enhanced stability criteria and conservatism comparisons," Communications in Nonlinear Science and Numerical Simulation, vol. 54, pp. 118135, 2018.

[29] H. Lu, W. Zhou, C. Duan, and X. Qi, "Delay-dependent robust $H \infty$ control for uncertain singular time-delay system with Markovian jumping parameters," Optimal Control Applications \& Methods, vol. 34, no. 3, pp. 296-307, 2013.

[30] W. Zhou, H. Lu, C. Duan, and M. Li, "Delay-dependent robust control for singular discrete-time Markovian jump systems with time-varying delay," International Journal of Robust and Nonlinear Control, vol. 20, no. 10, pp. 1112-1128, 2010.

[31] S. Xu and T. Chen, "Robust Ho control for uncertain stochastic systems with state delay," IEEE Transactions on Automatic Control, vol. 47, no. 12, pp. 2089-2094, 2002. 
[32] J. H. Lee, S. W. Kim, and W. H. Kwon, "Memoryless Ho controllers for state delayed systems," Institute of Electrical and Electronics Engineers Transactions on Automatic Control, vol. 39, no. 1, pp. 159-162, 1994.

[33] Q. Li, B. Shen, Y. Liu, and F. E. Alsaadi, "Event-triggered Ho state estimation for discrete-time stochastic genetic regulatory networks with Markovian jumping parameters and timevarying delays," Neurocomputing, vol. 174, pp. 912-920, 2016.

[34] S. L. Hu, D. Yue, H. P. Xie, and Z. P. Du, "Event-triggered $H_{\infty}$ stabilization for networked stochastic systems with multiplicative noise and network-induced delays," Information Sciences, vol. 299, pp. 178-197, 2015.

[35] D. Yue, E. Tian, and Q. Han, "A delay system method for designing event-triggered controllers of networked control systems," IEEE Transactions on Automatic Control, vol. 58, no. 2, pp. 475-481, 2013.

[36] D. V. Dimarogonas, E. Frazzoli, and K. H. Johansson, "Distributed event-triggered control for multi-agent systems," IEEE Transactions on Automatic Control, vol. 57, no. 5, pp. 1291-1297, 2012.

[37] X.-M. Zhang and Q.-L. Han, "Global asymptotic stability analysis for delayed neural networks using a matrix-based quadratic convex approach," Neural Networks, vol. 54, pp. 57-69, 2014.

[38] C.-K. Zhang, Y. He, L. Jiang, and M. Wu, "Stability analysis for delayed neural networks considering both conservativeness and complexity," IEEE Transactions on Neural Networks and Learning Systems, vol. 27, no. 7, pp. 1486-1501, 2016.

[39] S.-I. Niculescu, "On delay-dependent stability under model transformations of some neutral linear systems," International Journal of Control, vol. 74, no. 6, pp. 609-617, 2001.

[40] Z. Jinxian, W. Wu, C. Fujun, and Y. Fuwen, "Free-weighting matrices approach to $\mathrm{H} \infty$ filtering for a class of uncertain linear systems with time-varying delay," in Proceedings of the 2009 Chinese Control and Decision Conference (CCDC), pp. 127-177, IEEE Press, Guilin, China, 2009.

[41] J.-H. Kim, "Further improvement of Jensen inequality and application to stability of time-delayed systems," Automatica, vol. 64, pp. 121-125, 2016.

[42] A. Seuret and F. Gouaisbaut, "Wirtinger-based integral inequality: application to time-delay systems," Automatica, vol. 49, no. 9, pp. 2860-2866, 2013.

[43] H.-J. Yu, Y. He, and M. Wu, "Improved generalized $\mathrm{H}_{2}$ filtering for static neural networks with time-varying delay via freematrix-based integral inequality," Mathematical Problems in Engineering, vol. 2018, Article ID 5147565, 9 pages, 2018.

[44] P. Park, W. I. Lee, and S. Y. Lee, "Auxiliary function-based integral/summation inequalities: application to continuous/discrete time-delay systems," International Journal of Control, Automation, and Systems, vol. 14, no. 1, pp. 3-11, 2016.

[45] X.-M. Zhang and Q.-L. Han, "Event-triggered $H_{\infty}$ control for a class of nonlinear networked control systems using novel integral inequalities," International Journal of Robust and Nonlinear Control, vol. 27, no. 4, pp. 679-700, 2017.

[46] B. Shen, L. Sun, and Z. Zhao, "Wirtinger's inequality is applied to event-driven output control of networked control systems with Ho performance," in Proceedings of the 2018 Chinese Control And Decision Conference (CCDC), pp. 4472-4478, IEEE, Shenyang, June 2018. 


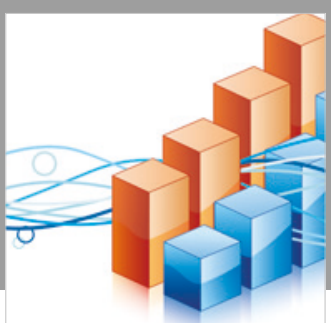

Advances in

Operations Research

\section{-n-m}
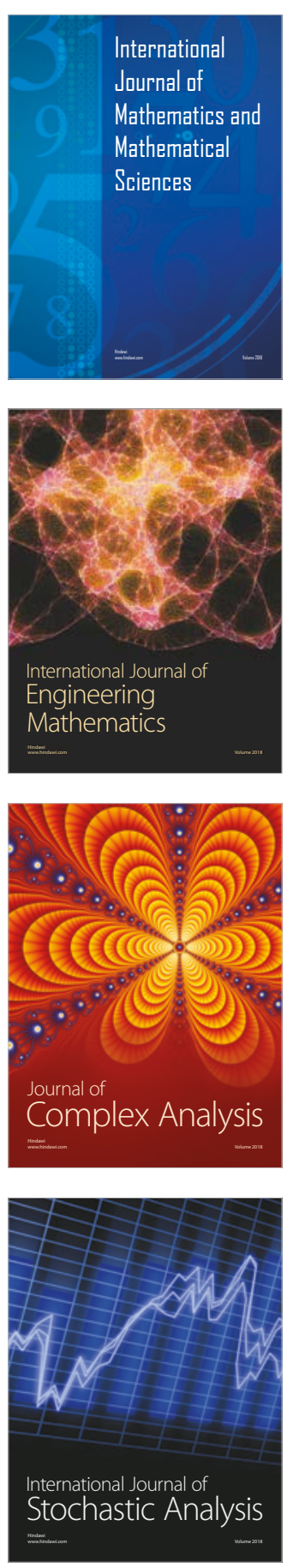
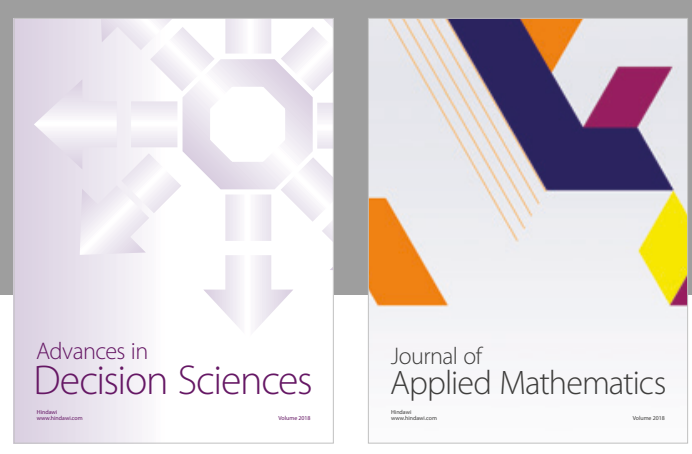

Journal of

Applied Mathematics
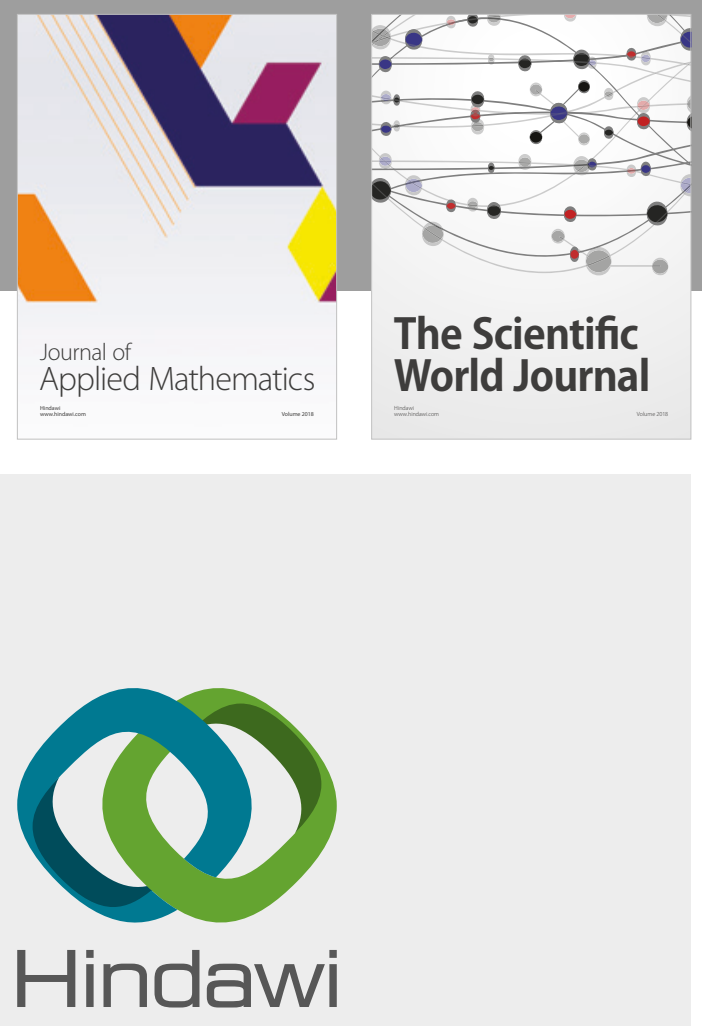

Submit your manuscripts at

www.hindawi.com

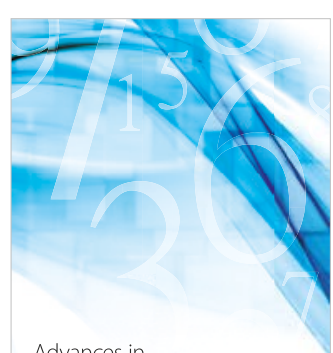

Advances in
Numerical Analysis
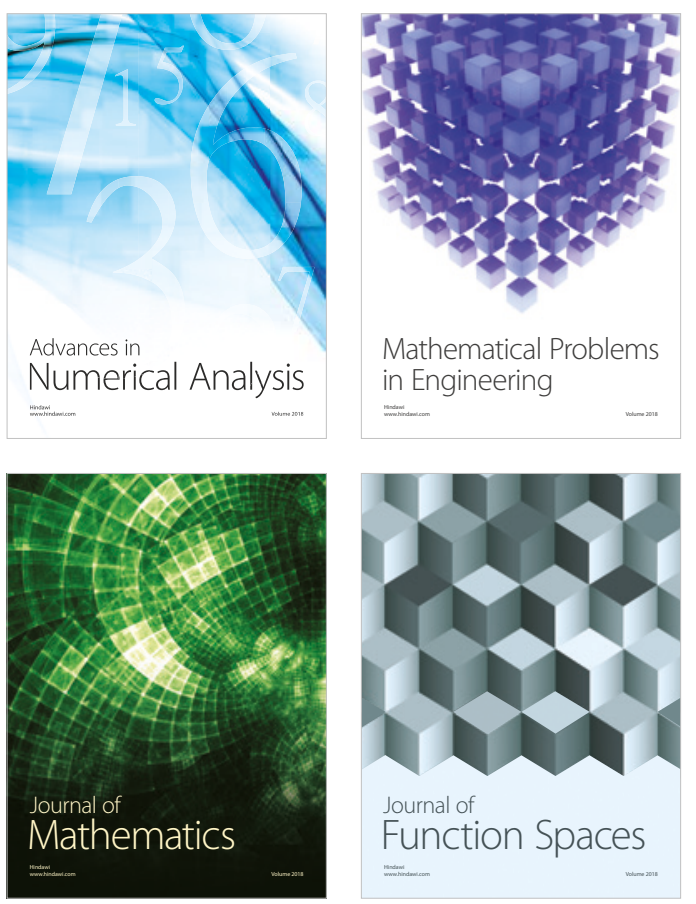

Mathematical Problems in Engineering

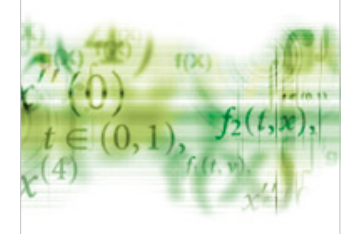

International Journal of

Differential Equations

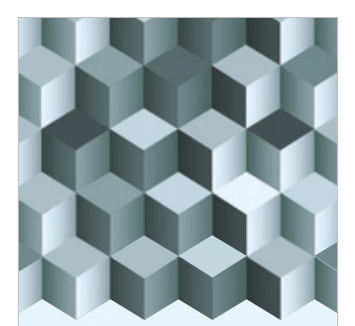

Journal of

Function Spaces
The Scientific

World Journal

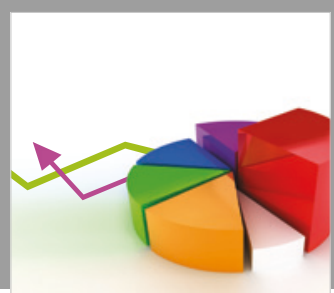

Journal of

Probability and Statistics
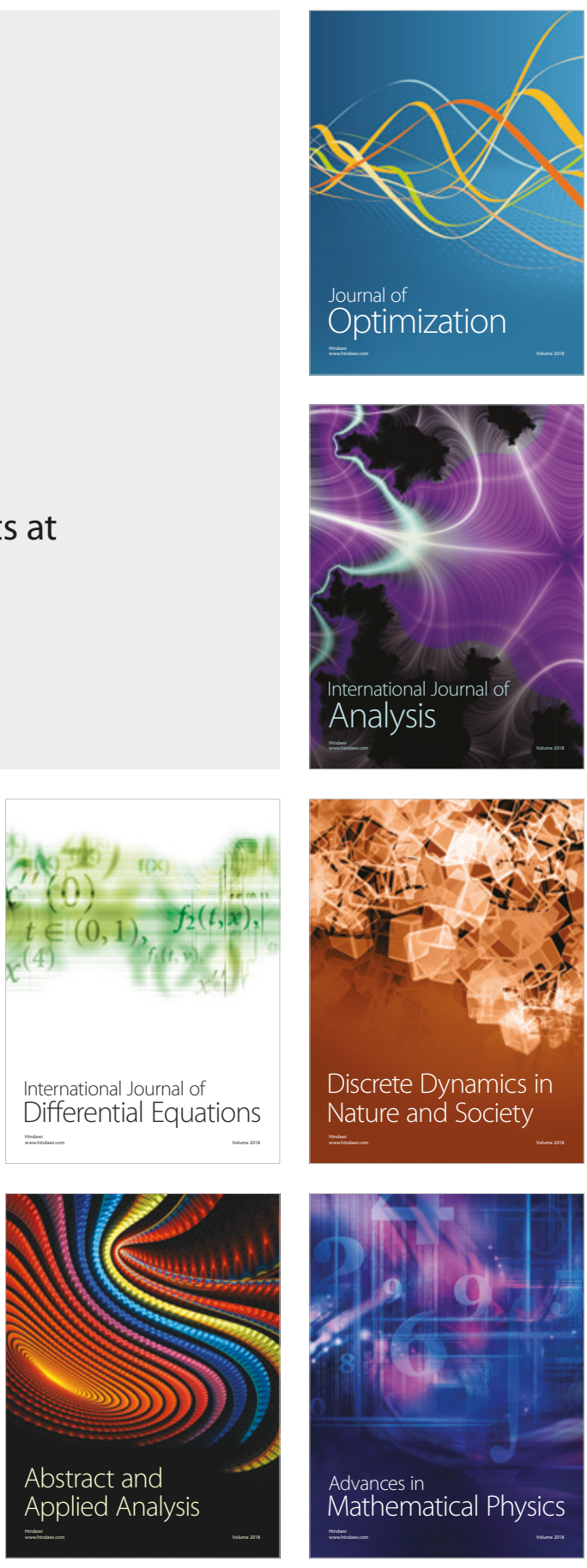